\title{
Parallel Neural Responses in Amygdala Subregions and Sensory Cortex during Implicit Fear Conditioning
}

\author{
J. S. Morris,* C. Buchel,* and R. J . Dolan*',1 \\ *Well come Department of Cognitive Neurology, Queen Square, London WC1N 3BG, United Kingdom; and †Royal Free \\ and University College Hospitals School of Medicine, Rowland Hill Street, London NW3 2DF, United Kingdom
}

Received August 11, 2000

We used event-related fMRI to measure neural activity in volunteer subjects during acquisition of an implicit association between a visual conditioned stimulus (CS+) (angry face) and an auditory unconditioned stimulus (UCS) (aversive, loud noise). Three distinct functional regions were identified within left amygdala: a UCS (noise)-related lateral region, a CS+-related ventral region, and a dorsal region where CS+related responses changed progressively across the learning session. Differential neural responses to the visual CS+ were also evoked in extrastriate and auditory cortices. Our results indicate that learning an association between biologically salient stimuli of different sensory modalities involves parallel changes of neural activity in segregated amygdala subregions and unimodal sensory cortices. 2001 Academic Press

\section{INTRODUCTION}

The amygdaloid complex is a heterogeneous structure composed of at least 13 different subnuclei with complex intrinsic and extrinsic connections (Amaral et al., 1992). The major input region of the amygdala is the lateral nucleus, which receives convergent afferents from all sensory modalities (Amaral et al., 1992). The lateral nucleus itself projects to medial and basal nuclei, where additional extrinsic inputs from other functional systems produce further modification of neural signals (Pitkanen et al., 1997). Intrinsic projections from medial and basal nuclei converge onto the central nucleus, which, in turn, sends extrinsic efferents to various subcortical structures controlling autonomic activity (Amaral et al., 1992; Pitkanen et al., 1997). Other amygdaloid subnuclei project widely to occipital, temporal, prefrontal, and cingulate cortices (Amaral et al., 1992). This anatomical connectivity makes the amygdaloid complex ideally placed both to

${ }^{1}$ To whom correspondence should be addressed. integrate sensory signals from different modalities and to influence behavioral responses.

Experiments in both animals and humans have implicated the amygdala in aversive (fear) conditioning, i.e., in the ability of a neutral stimulus to elicit conditioned responses as the result of an association with an aversive unconditioned stimulus (UCS) (Davis, 1992; LeDoux, 1995; Buchel et al., 1998; LaBar et al., 1998). Human neuroimaging studies using backward masking have also shown that the amygdala can respond to behaviorally salient visual stimuli that are outside subjects' explicit awareness (Whalen et al ., 1998; Morris et al., 1998c). However, although the amygdala has been shown to respond to "unseen" masked stimuli on the basis of their learned behavioral significance (Morris et al ., 1998c), there are no previous data concerning amygdala involvement in the acquisition of learning without awareness. Indeed, there has been controversy about whether learning without awareness can occur at all (Ohman et al., 2000). Nevertheless, behavioral studies suggest that stimulus-stimulus associations can be formed implicitly (i.e., without subjects' awareness) when the stimuli involved are biologically salient, e.g., snakes, spiders, or angry faces (Esteves et al., 1994).

We used event-related functional magnetic resonance imaging (fMRI) to measure neural responses during acquisition of an implicit association between a biologically salient visual stimulus (an angry face) and an aversive auditory stimulus (a loud noise). Two target faces (CS + and CS - , both with angry expressions) were presented to healthy volunteers during scanning. Angry faces were specifically chosen since they are optimal stimuli for this type of implicit learning (Ohman et al., 2000; Esteves et al., 1994). Half the presentations of both CS + and CS - were backwardly masked with neutral faces, to ensure that subjects had no explicit awareness of half the targets. During these masked presentations, the CS + was always immediately followed by a 100-dB noise (UCS). The noise UCS was never paired with the other target face (CS-) or with unmasked CS + (Fig. 1). Consequently, the proto- 
a
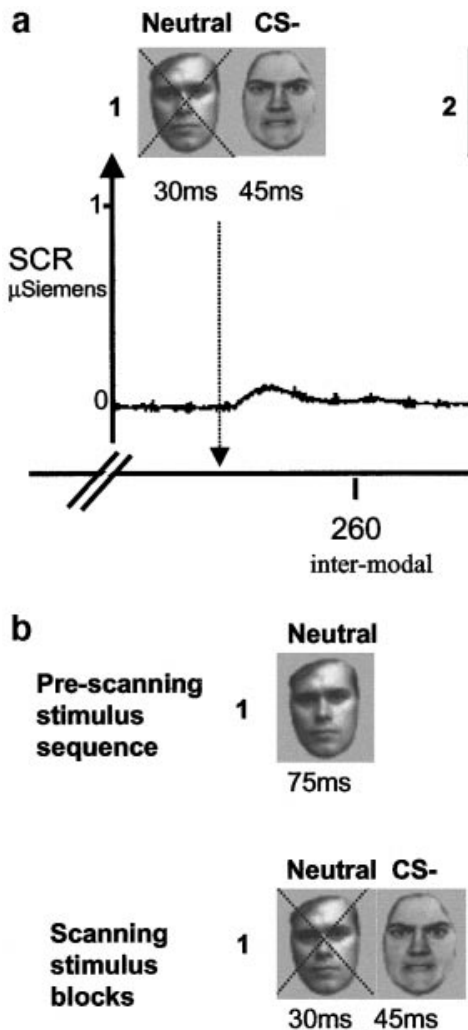

b
Pre-scanning

$30 \mathrm{~ms} 45 \mathrm{~ms}$
Neutral CS+

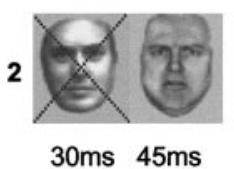

CS+ Mask UCS

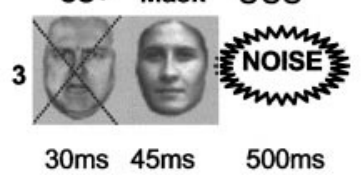

cs- Mask

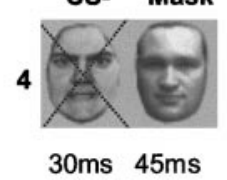

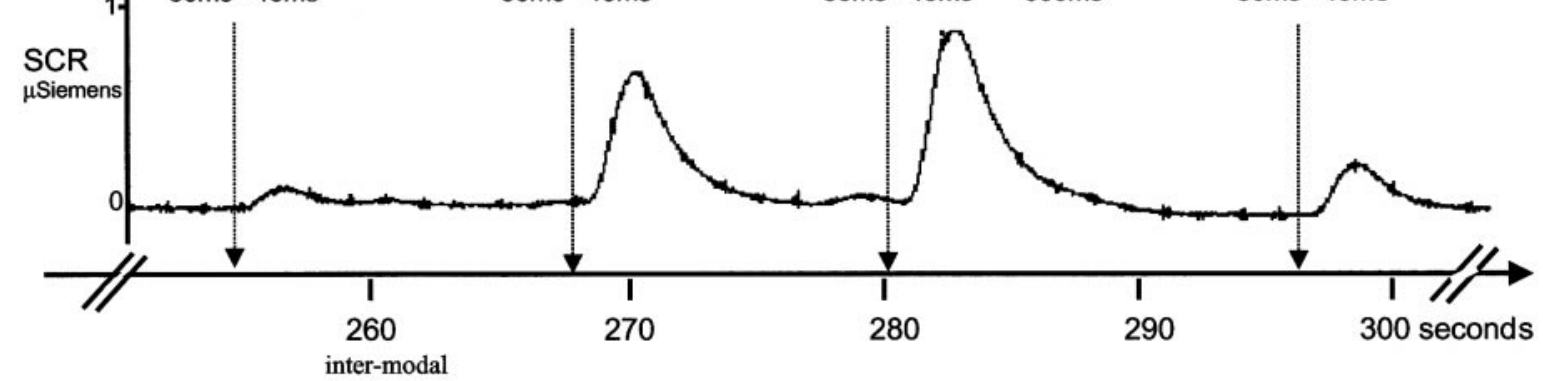
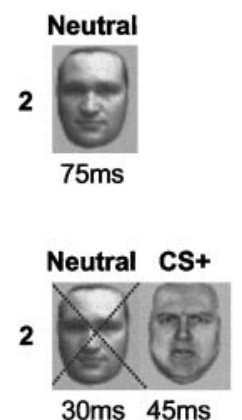
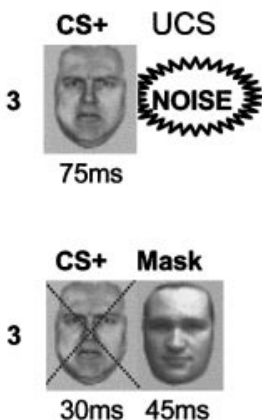
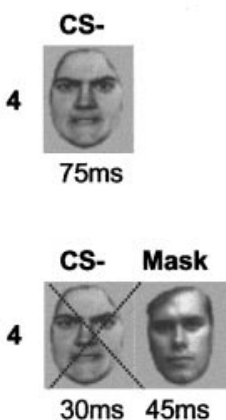

FIG. 1. Experimental design. (a) The four event types, i.e. (1) unmasked CS-, (2) unmasked CS+, (3) masked CS+/noise UCS, and (4) masked CS-, are illustrated, together with a corresponding trace of skin conductance responses (SCRs) acquired from one representative subject during scanning. Due to the presence of the mask, the first face presented in each pair (indicated by crosses) was not explicitly perceived by subjects. Stimulus durations are indicated in milliseconds, whereas time elapsed from the beginning of the scanning session is shown in seconds. The onset of each stimulus complex is indicated by arrows. SCR amplitude is shown in microsiemens. (b) The experimental design of a previous PET study (M orris et al., 1998c) is shown for comparison. Pairing of the CS + face and the noise UCS occurred explicitly (i.e., without masking) during a prescanning sequence. Masked faces were presented in condition blocks during each 90-s PET scan.

col ensured that the CS+/UCS stimulus association was implicit. Throughout the experiment, subjects' explicit task was to report (via button presses) any perception, however fleeting, of the target faces. A randomized, mixed-trial design was used for the various face and masking conditions, and the identity of the CS + face was balanced across subjects to ensure that the unmasked CS + and CS - conditions were identical except for the pairing of CS + and UCS during masked presentations. Subjects' skin conductance responses (SCRs) were measured throughout the experiment to index autonomic conditioning.

\section{METHODS}

\section{Subjects}

Six right-handed subjects (four male, two female, mean age 29.3 years) were recruited by advertisement. Subjects had no history of neurological or psychiatric problems and were not taking any medication at the time of the study. All subjects gave informed consent and the study was approved by thelocal hospital ethics committee.

\section{Data Acquisition}

Neuroimaging data were acquired with a 2-T Magnetom VISION whole-body MRI system equipped with a head volume coil. Contiguous multislice T2* weighted echoplanar images were obtained using a sequence that enhanced blood oxygenation level-dependent (BOLD) contrast. Volumes covering the whole brain (48 slices; slice thickness $2 \mathrm{~mm}$ ) were obtained every $4.1 \mathrm{~s}$. A T1-weighted anatomical MRI was also acquired for each subject.

Throughout scanning, SCRs from all six subjects were monitored to index autonomic conditioning. Data from one subject was unfortunately lost due to technical problems. SCRs were measured with Biodata galvanic skin response equipment using $\mathrm{Ag} / \mathrm{AgCl}$ electrodes attached to the palmar surface of the middle phalanges of the index and middle fingers of the left hand. Readings of skin conductance were sampled at $100 \mathrm{~Hz}$ and stored digitally on computer. Using the SCR in the 4-s period prior to presentation as a baseline, the maximal SCR deflection in the period 0.5- $4 \mathrm{~s}$ following a face presentation was assigned as the value for the SCR to that face. The data were square root 
transformed to attain statistical normality, prior to calculating means and standard errors for each condition. The time course of conditioning was assessed by dividing each session, for each condition, into five epochs of five trials (omitting the first trial). Condition means and standard errors were calculated for each epoch (Fig. 2).

\section{Experimental Design}

Immediately prior to scanning, subjects were shown pictures of two angry faces. Subjects' explicit task during the scanning session was to indicate, via a button press, any subsequent occurrence of these target stimuli, however fleeting. For each stimulus presentation, subjects pressed one button with their right middle finger to indicate that they had seen a target and another button with their index finger to indicate that they were not aware of a target. During scanning, stimuli were presented according to four different event types (Fig. 1): (1) One of the angry faces (CS-) was shown for $45 \mathrm{~ms}$, preceded by a $30-\mathrm{ms}$ neutral face. (2) The other angry face (CS+) was also shown for 45 ms, preceded by a 30-ms neutral face. (3) The CS + face was shown for $30 \mathrm{~ms}$, followed immediately by a 45-ms neutral face and a 500-ms 100-dB white noise burst (UCS). (4) The CS - face was shown for $30 \mathrm{~ms}$ and followed by a 45-ms neutral face. Twenty-six replications of each event type were presented in a randomized order. The UCS always occurred in event type 3, but never in any other event type. In order to prevent conditioning to the mask, 18 different neutral faces were used as masks for each session, in a random order of presentation. Angry faces were used as targets since behavioral studies have shown them to be optimal stimuli for masked conditioning (Esteves et al., 1994). The interstimulus interval was randomly varied between 2.5 and 3.5 TRs (i.e., 10.25-14.35 s) to ensure that event onsets were evenly distributed in time across image slices.

\section{Data Analysis}

The fMRI data were analyzed using statistical parametric mapping (Friston et al., 1995) (see also http:// www.fil.ion.ucl.ac.uk/spm). Following realignment to the first volume, the functional (T2*-weighted) scans were spatially normalized to a standard template. The structural (T1-weighted) MRI s were coregistered to the functional scans and transformed into the same standard space. The functional data were smoothed using a 6-mm (full width at half-maximum) isotropic Gaussian kernel to allow for corrected statistical inference. The evoked hemodynamic responses for the four different stimulus events were modeled as $\delta$ functions convolved with a synthetic hemodynamic response function and its temporal derivative (J osephs et al., 1997).
Specific effects (e.g., unmasked CS+ > unmasked CS-) were tested by applying linear contrasts to the parameter estimates for each event. The resulting $t$ statistic at every voxel constitutes an SPM. Reported P values are corrected for the number of comparisons made within each a priori region of interest, i.e., amygdala, specific thalamic nuclei (pulvinar and MGB), fusiform gyrus, and auditory cortex (Worsley et al., 1996). An activation in right occipital cortex $(x=32, y=-88$, $z=20$ ) is also reported because although not predicted a priori, the magnitude of the effect in this region survived correction for comparisons across the entire brain. A time-by-event interaction analysis was performed by multiplying the regressors for the stimulus events with a mean corrected exponential function having a time constant one-quarter of the session length. Contrasts tested for the difference of the interaction terms between the unmasked CS+ and unmasked CS - conditions. The data were analyzed for all six subjects individually, and also as a group, modeling evoked responses in a subject-specific way. Due to the limited number of subjects, a fixed-effects, rather than random-effects, statistical model was used for the group analysis of the data. While the results of the fixed-effects group analysis considered al one have limited generalizability, the individual analyses identified similar responses for each reported activation in all of the six subjects.

\section{RESULTS}

Only $1.9 \%$ of masked targets were reported, compared to $95.1 \%$ of unmasked targets. On subsequent debriefing, five of the six subjects showed no explicit knowledge of the masking procedure, while the remaining subject reported occasional awareness of "flickering" and "superimposed" faces. None of the six subjects exhibited any explicit knowledge about the association between the angry CS + face and the UCS. Despite this lack of awareness about the behavioral significance of the CS+, subjects exhibited significantly increased $(P<0.05)$ mean skin conductance responses to the unmasked CS + face, compared to the CS - (Fig. 2), indicating that implicit associative learning had occurred. Enhanced SCRs also occurred in the masked CS+/UCS(noise) condition (Figs. 1a and 2). However, the 75-ms asynchrony between the masked $\mathrm{CS}+$ and the UCS is outside the resolution of the SCR data, and thus the relative contribution of each of the stimulus components to the evoked autonomic response cannot be determined.

We first identified neural responses evoked by the aversive noise (UCS) by contrasting the UCS condition with the three other conditions in which the noise was absent. This contrast revealed enhanced responses in bilateral medial geniculate nucleus (MGN) (left, $x=$ $-20, y=-24, z=-4, P<0.001$, corrected; right, $x=$ 
a

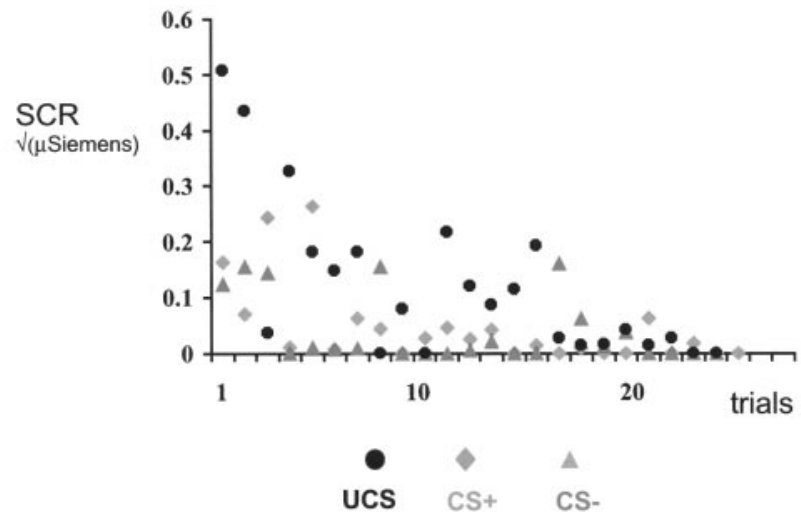

b

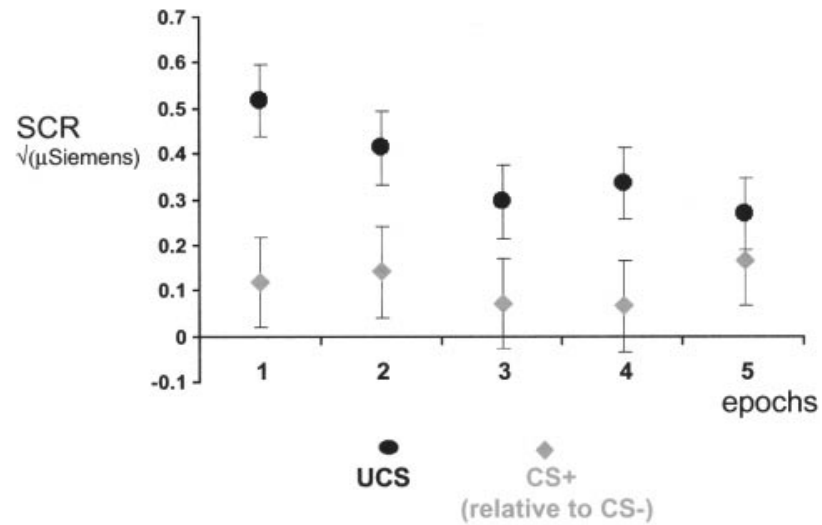

FIG. 2. Skin conductance responses. (a) Plot of SCR amplitudes for every trial in one scanning session. SCR amplitude is shown in $\sqrt{ }$ (microsiemens). (b) Mean SCR amplitudes for five of the six subjects. Each epoch represents five consecutive trials. CS+ SCR amplitudes are plotted relative to CS - amplitudes. Error bars represent 1 standard error. SCR amplitude is shown in $\sqrt{ }$ (microsiemens).

$28, y=-20, z=-4, P<0.05$, uncorrected), bilateral auditory cortex (left, $x=-52, y=-24, z=6, P<$ 0.001 , corrected; right, $x=54, y=-22, z=8, P<0.05$, corrected), and left lateral amygdala ( $\mathrm{x}=-30, \mathrm{y}=$ $-10, z=-10, P<0.05$, corrected) (Fig. 3). Critically, UCS (noise)-evoked responses in lateral amygdala were constant across the learning session (i.e., did not show learning-related habituation or augmentation). No differential responses to the face stimuli (CS + and CS-) were identified in the UCS-related lateral amygdala region (Fig. $3 d$ ).

We next identified brain regions with enhanced re sponses to the CS + face by contrasting neural activity evoked by the CS + alone (i.e., unmasked and without noise) with that elicited by the unmasked CS-. Increased CS + responses were observed in right occipital cortex $(x=32, y=-88, z=20, P<0.001$, corrected), right pulvinar nucleus of the thalamus $(x=6, y=-22$, $z=14, P<0.05$, corrected), and, crucially, in a left ventral amygdala region that extended into adjacent medial cortex $(x=-12, y=-8, z=-26, P<0.05$, corrected) (Fig. 4a). In contrast to findings of previous conditioning experiments (Buchel et al., 1998; LaBar et al., 1998), CS +-evoked responses in this ventral region were sustained (i.e., did not progressively increase or decrease) throughout the learning session (Fig. 4b).

In our final analysis we addressed the question of time-dependent changes associated with learning by modeling the neuroimaging data with an exponential function. This analysis identified a dorsal region of left amygdala ( $\mathrm{x}=-18, \mathrm{y}=2, \mathrm{z}=-14, \mathrm{P}<0.05$, corrected) where CS+-evoked responses were greater than CS- responses in the initial stages of learning (Figs. 5a and 5b). However, as the session continued, responses elicited by the CS+ progressively decreased (relative to $\mathrm{CS}-$ ), leading eventually to a reversal of differential activity, i.e., overall responses were greater to $\mathrm{CS}$ - than to CS+ (Fig. 5b). The same analysis revealed a region of left auditory cortex $(x=-44, y=$ $-18, z=12, P<0.05$, corrected) with a similar pattern of time-dependent activity (Figs. $4 \mathrm{c}$ and $4 d$ ). N ote that left auditory cortex also showed enhanced responses to the noise UCS (see above). In contrast, bilateral regions of fusiform gyrus (right, $x=36, y=-62, z=-2$, $\mathrm{P}<0.05$, corrected; left, $\mathrm{x}=-32, \mathrm{y}=-66, \mathrm{z}=-2, \mathrm{P}<$ 0.001 , corrected) showed an opposite pattern of response, i.e., progressive, time-dependent increases in CS+-evoked responses (Figs. 4c and 4d). This response profile was expressed without a significant mean difference in CS + and CS - activity.

\section{DISCUSSION}

The results of the present study represent the first neuroimaging evidence for learning-related functional segregation within the human amygdala. Three distinct responses were identified in subregions of the amygdaloid complex: a sustained UCS-evoked response in lateral amygdala; a sustained CS+-evoked response in ventral amygdala that extended into overlying cortex; and a time-dependent CS+-related response in dorsal amygdala that extended anteriorly into basal forebrain. Although the resolution of our neuroimaging data prevents attribution of these differential responses to specific amygdala subnuclei, the segregated activity observed in the amygdaloid complex is in general accord with the anatomical and functional heterogeneity of this structure as revealed in animal studies (Amaral et al., 1992; Pitkanen et al., 1997).

The precise nature of the associative plasticity involved in fear conditioning is currently a matter of controversy, with conflicting views as to whether the amygdala represents a primary locus of learning-related synaptic changes (Fansel ow and LeDoux, 1999; Cahill et al., 1999). Although the present study is unable to resolve this debate, it does provide evidence for specialization of learning-related processing within the amygdaloid complex itself. For example, the lateral subregion of the amygdala, which had increased re- 
a

\section{medial geniculate}
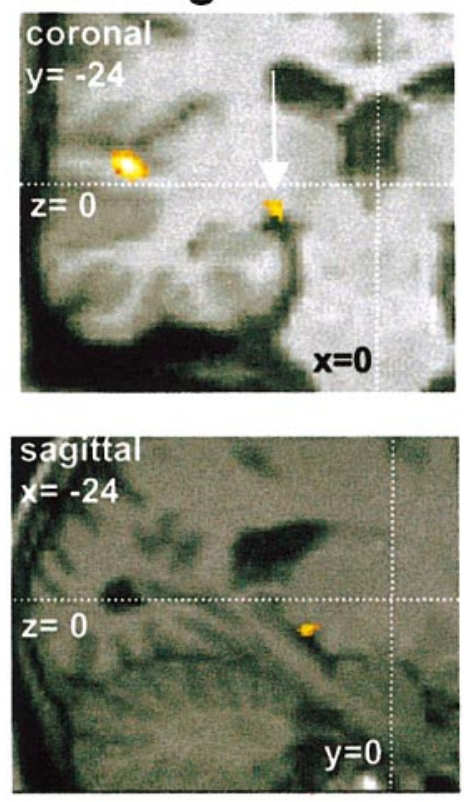

b
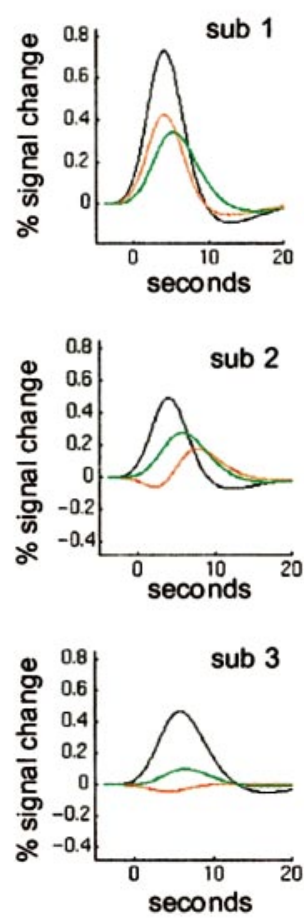

C

\section{lateral amygdala}
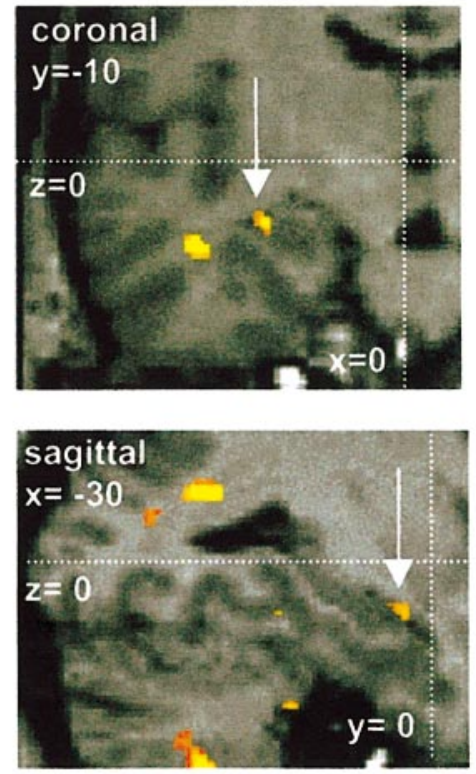

d
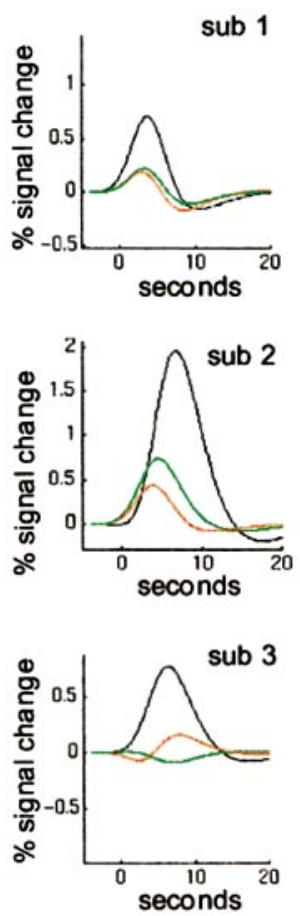

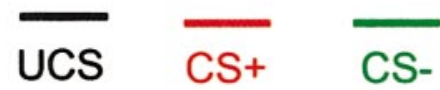

FIG. 3. Increased neural responses evoked by the UCS. Statistical parametric maps of increased neural responses to the masked CS+/UCS stimulus (relative to the three other conditions) in (a) left medial geniculate nucleus and (c) left lateral amygdala. The lateral amygdala region is indicated by arrows. Group activations are displayed on coronal and sagittal sections of a normalized MRI from an individual subject. Plots of the hemodynamic responses evoked by the masked CS+/UCS, unmasked CS+, and unmasked CS - events are shown for three individual subjects in (b) left medial geniculate and (d) left lateral amygdala. Responses are fitted to a canonical hemodynamic response function and its temporal derivative.

sponses in the UCS (noise) condition, did not exhibit differential responses to the unmasked CS+ and CSfaces (Figs. 3c and 3d). This response profile suggests that lateral amygdala has a greater involvement in sensory processing of the noise UCS than in associative processes related to face-noise pairings. Anatomical and electrophysiological data obtained in rats indicate that the amygdala not only receives a direct anatomical connection from auditory thalamus (MGN) but also possesses auditory-responsive cells (Bordi and LeDoux, 1992).

In ventral amygdala, in contrast, increased re sponses were specifically evoked by the CS + face. These CS+-related activations did not progressively increase or decrease during the learning session, suggesting that differential responses in ventral amygdala were rapidly established at the start of learning and then maintained throughout the experiment. This response profile contrasts with neuroimaging data from previous conditioning studies, which have consistently shown progressive decreases in amygdala CS+ responses during the learning session (Buchel et al., 1998; LaBar et al., 1998). The reason for these divergent findings is not clear. The sustained ventral response may be linked to our use of biologically salient or "prepared" stimuli. Since angry faces are known to facilitate both the acquisition and the persistence of learned associations (Esteves et al., 1994), these "prepared" stimuli, not used in previous studies (Buchel et al., 1998; LaBar et al., 1998) may have played a critical role in the pattern of amygdala activity seen in the present experiment. An equally important consideration is our novel use of an implicit learning paradigm, suggesting that task-related factors may be important in modulating responses in the human amygdala.

In dorsal amygdala, on the other hand, a striking reversal in conditioning-related responses was seen during learning, i.e., although $\mathrm{CS}+>\mathrm{CS}-$ responses 
a

\section{ventral amygdala}

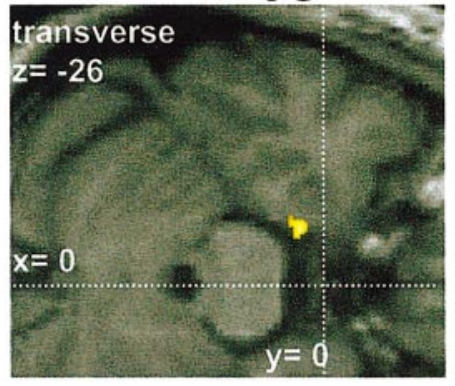

4

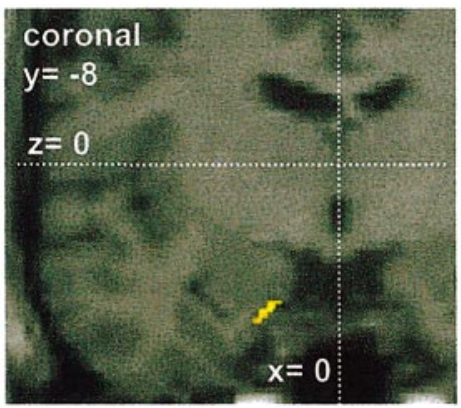

b
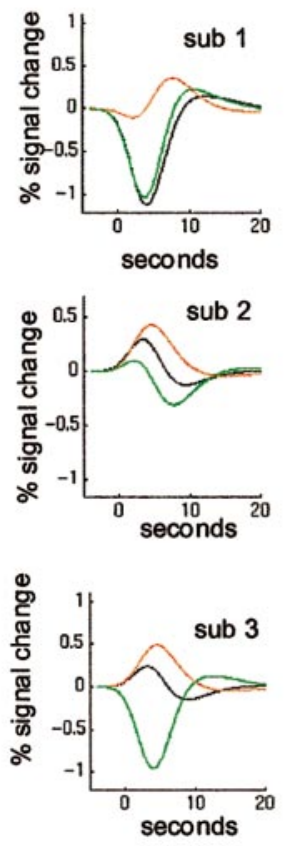

UCS c

\section{fusiform gyrus}
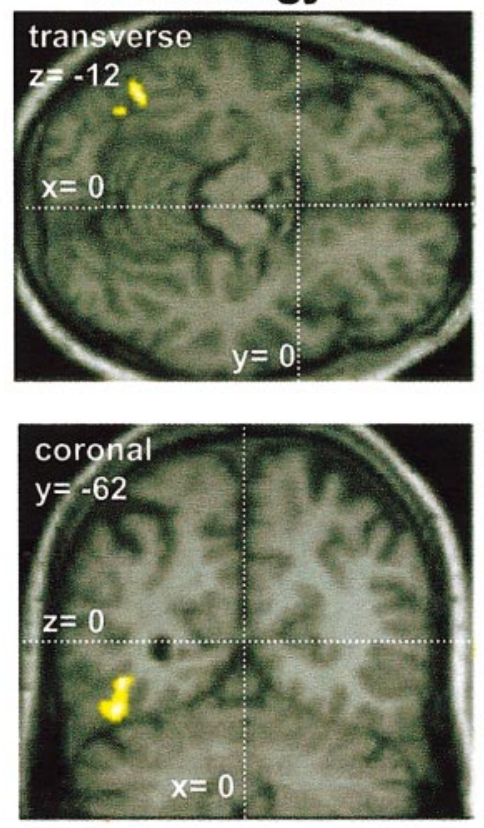

CS+ d

CS+
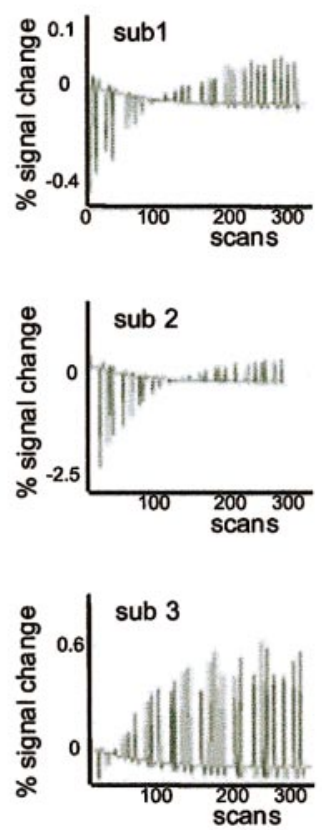

a

\section{dorsal amygdala}

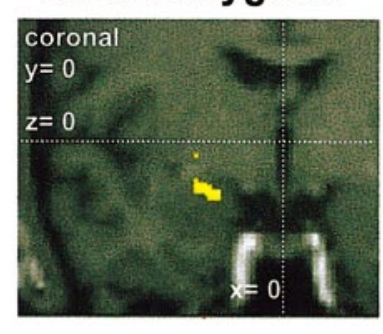

5

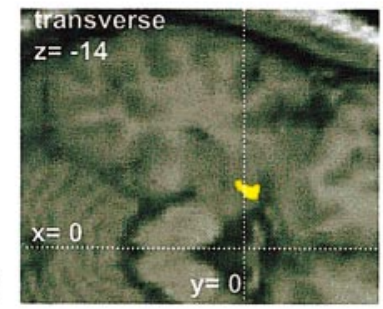

\section{CS+}
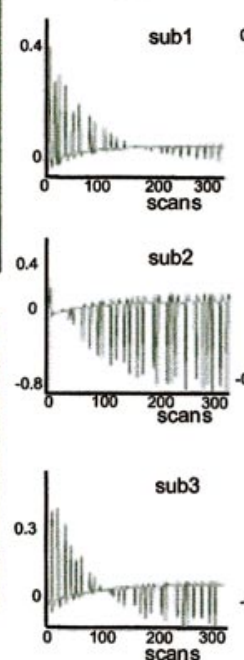

CS-

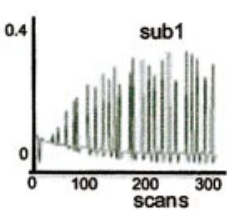

sub2
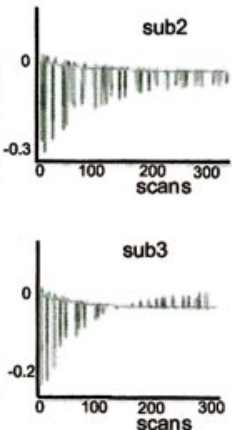

c auditory cortex
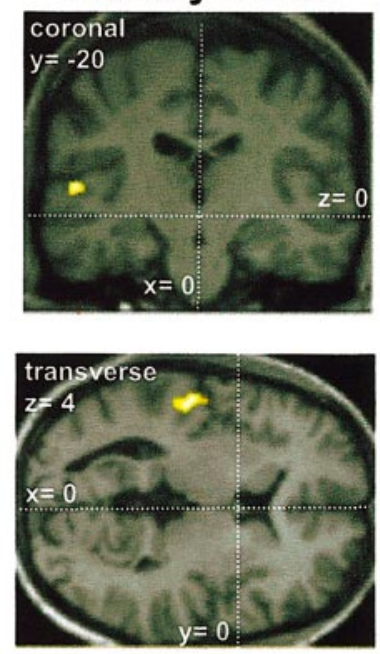

d

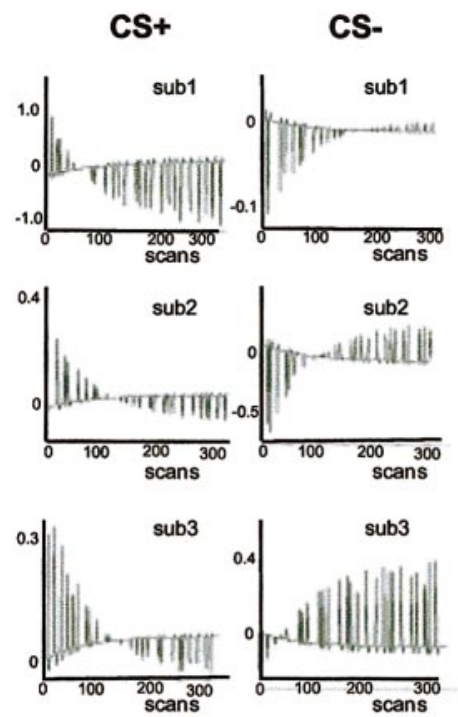

FIG. 4. Increased neural responses evoked by the CS+. (a) An SPM showing increased neural responses to the unmasked CS+ face (relative to unmasked CS-) for left ventral amygdala. (b) Plots of responses in left ventral amygdala evoked by the masked CS+/UCS, unmasked CS+, and unmasked CS - event types are shown for three individual subjects. Responses are fitted to a canonical hemodynamic response function and its temporal derivative. (c) An SPM showing exponentially increasing responses across time to the unmasked CS + (relative to unmasked CS-) in left fusiform gyrus. (d) Plots of the change in CS+ evoked responses in left fusiform gyrus across the scanning session are shown for the same subjects as in (b). The responses are fitted to an exponential function with a time constant one-quarter of the session length. In both (a) and (c), group activations are displayed on transverse and coronal sections of a normalized MRI from an individual subject.

FIG. 5. Decreased neural responses evoked by the CS+. SPMs of decreased neural responses to the CS + face (relative to CS-) in (a) left dorsal amygdala and (c) left auditory cortex are shown. Group activations are displayed on transverse and coronal sections of a normalized MRI from an individual subject. Plots of changes in hemodynamic response for unmasked CS + and unmasked CS - event types are shown for three individual subjects in (b) left dorsal amygdala and (d) left auditory cortex. Responses are fitted to an exponential function with a time constant one-quarter of the session length. 
were observed initially, a CS $->C S+$ pattern of re sponse was evident by the end of the session (Fig. 5). As a consequence of these time-dependent changes, opposing neural responses emerged in different subregions of the amygdala during learning, i.e., increased CS+ responses in ventral amygdala and decreased CS + re sponses in dorsal amygdala (Figs. 4a, 4b, 5a, and 5b). Interestingly, animal electrophysiological studies have also demonstrated reciprocal changes in firing probability between different amygdala subregions (Collins and Pare, 1999). It has been proposed that these reciprocal response patterns result from changes in parallel, excitatory, and inhibitory connections between amygdala subnuclei (Collins and Pare, 1999). It is possible, therefore, that the progressive "polarization" of ventral and dorsal amygdala responses in the present study may reflect the occurrence of similar associative changes in intra-amygdala circuits. However, in view of the amygdala's extensive extrinsic anatomical connections (Amaral, 1992), external modulatory factors may also play an important role.

Previous neuroimaging studies have shown that the fusiform gyrus mediates face-selective responses (Haxby et al., 1994). The progressive increase in fusiform responses to the CS+ face (Figs. 4c and 4d) suggests, therefore, that processing of the CS+ face is selectively enhanced as it becomes more emotionally salient with learning, consistent with predictions from neuropsychological theories of emotion (Damasio, 1999). The absence of a significant mean differential response, on the other hand, may reflect the fact that CS + and CS - faces elicit similar fusiform activations at the beginning of the session. Thus, although CS+ fusiform responses increase progressively with time, the increase in activation is not sufficient to produce a significant mean difference from the CS- condition when the whole session is considered.

Since fusiform gyrus and occipital cortex do not receive direct auditory inputs, the learning-related changes in their activity must depend on extrinsic modulatory influences from brain areas that do process the noise UCS. The precise nature of this modulation cannot be determined from our current neuroimaging data. However, it is striking that responses to both UCS and CS+ were identified within segregated regions of the amygdala (Figs. 3c, 3d, 4a, and 4b), a structure that is known to send extensive projections back to early regions of visual cortex (Amaral et al., 1994). The parallel learning-related responses observed in ventral amygdala and visual cortex (Fig. 3) are consistent with a proposal, therefore, that "feedback" efferents from basal amygdala nuclei mediate emotion-dependent modulation of visual processing (Amaral et al., 1994; Morris et al., 1998a).

The implicit association of the CS+ face and the noise UCS also resulted in learning-dependent modulation of auditory cortex activity (Figs. $4 c$ and $4 d$ ).
Intriguingly, despite the absence of a direct visual input to auditory cortex, the CS + face (a purely visual stimulus) evoked auditory responses that progressively decreased (relative to $\mathrm{CS}_{-}$) ) during the learning session (Fig. 4d). These data accord with autoradiographic data obtained in rats showing that a visual stimulus previously paired with a tone and foot shock also el icits relative decreases in auditory cortex activity (Cahill et al., 1996). Although the neural mechanisms underlying this phenomenon are uncertain, previous animal and human experiments indicate that the amygdala may have a critical role (Weinberger, 1995; Armony et al., 1998; Morris et al., 1998b). In the present study, the segregated visual CS + and auditory UCS responses in ventral and lateral amygdala (Figs. 3 and 4), and the parallel time-dependent activity in dorsal amygdala and auditory cortex (Fig. 5), also suggest amygdala involvement in this form of intermodal emotional learning.

Some learning theorists have proposed that when one stimulus becomes associated with a second stimuIus, the first may be able to activate a "representation" of the second in relevant sensory cortex (Konorski, 1967). Data from an explicit associative learning experiment (Mclntosh et al., 1998) in which an auditory tone predictive of a light flash came to elicit responses in human visual cortex, accord with this proposal. However, a possible interpretation of this earlier finding is that the modulation of visual cortex reflects changes in subjects' selective attention or conscious imagery, rather than associative learning. The implicit conditioning paradigm employed in the present study excludes an attentional explanation for the observed sensory modulation, since subjects had no explicit awareness of the stimulus association.

Other learning theorists have proposed that an automatic shift of processing resources occurs from UCS to $\mathrm{CS}+$ during classical conditioning as the CS+ becomes a reliable "predictor" of the UCS (Wagner, 1979). This proposal is supported by animal el ectrophysiological data demonstrating that primary auditory cortex responses to an auditory CS become more likely as a result of conditioning-related shifts in the tuning curves of auditory cells (Wenberger, 1995). Our results, showing that progressive increases in fusiform gyrus responses to the CS + face during learning (Fig. 4) are accompanied by progressive decreases in auditory cortex responses (Fig. 5), are also consistent with this conjecture.

It is important to note that whereas CS + and UCS pairings occurred during masking (i.e., the association was acquired outside subjects' awareness), learningrelated responses to $\mathrm{CS}+$ and $\mathrm{CS}$ - faces were measured during unmasked (i.e., explicitly reported) presentations. This paradigm contrasts, therefore, with a previous neuroimaging study (Morris et al., 1998c) in which CS+ and UCS faces were paired explicitly (i.e., 
without masking) prior to scanning and neural activity was measured during subsequent masked and unmasked presentations, with the noise UCS omitted (Fig. 1b). I n the previous study (Morris et al., 1998c), as in the present experiment, unmasked CS+ faces evoked differential responses in left amygdala. However, whereas masked CS+ faces elicited increased responses in right amygdala in the previous study, the masked CS + and UCS pairings in the present experiment did not evoke similar right amygdala activity. There are several important methodological differences between the two studies that may explain these contrasting results. First, the noise UCS always followed the masked CS + in the present experiment, but never followed the masked CS + in the previous study (Fig. 1). Second, in the present mixed-trial, event-related, BOLD fMRI study, responses were measured during conditioning, whereas in the previous positron emission tomography experiment (Morris et al ., 1998c), subjects were scanned after conditioning with blocked presentation of stimuli (Fig. 1). It is possible, therefore, that the presence or absence of the UCS, or other differences in the learning paradigms, may have produced different patterns of right amygdala activity.

In conclusion, the present study provides the first demonstration of learning-related functional heterogeneity within the human amygdala. Although the spatial resolution of the current data allow the identification only of subregional differences in activity rather than individual nuclei, the results suggest that mapping distinct functional subsystems within the human amygdala may be possible using neuroimaging techniques with high spatial resolution. The parallel changes in amygdala and sensory cortex responses observed in the present study also provide novel evidence about how biologically salient stimulus-stimulus associations are mediated in the brain. The results suggest that human emotional learning, even when acquired without explicit awareness, involves changes in both intrinsic amygdala responses and extrinsic amygdala connectivity.

\section{ACKN O WLEDGMENT}

J .S.M., C.B., and R.J .D. are supported by the Wellcome Trust.

\section{REFERENCES}

Amaral, D. G., Price, J . L., Pitkanen, A., and Carmichael, S. T. 1992. Anatomical organization of the primate amygdaloid complex. In The Amygdala: Neurobiological Aspects of Emotion, Memory and Mental Dysfunction (J. P. Aggleton, Ed.), pp. 1-66. Wiley-Liss, New York.

Armony, J. L., Quirk, G. J., and LeDoux, J. E. 1998. Differential effects of amygdala lesions on early and late plastic components of auditory cortex spike trains during fear conditioning. J . Neurosci. 18: 2592-2601.

Bordi, F., and LeDoux, J . E. 1992. Sensory tuning beyond the sensory system: An initial analysis of auditory properties of neurons in the lateral amygdaloid nucleus and overlying areas of striatum. J . Neurosci. 12: 2493-2503.

Buchel, C., Morris, J ., Dolan, R. J ., and Friston, K. J . 1998. Brain systems mediating aversive conditioning: An event-related fMRI study. Neuron 20: 947-957.

Cahill, L., Ohl, F., and Scheich, H. 1996. Alteration of auditory activity with a visual stimulus through conditioning: A 2-deoxyglucose analysis. Neurobiol. Learn. Memory 65: 213-222.

Cahill, L., Weinberger, N. M., Roozendaal, B., and McGaugh, J . L. 1999. Is the amygdala a locus of "conditioned fear"? Some questions and caveats. Neuron 23: 227-228.

Collins, D. R., and Pare, D. 1999. Reciprocal changes in the firing probability of lateral and central medial amygdala neurons. J . Neurosci. 19: 836-844.

Damasio, A. 1999. TheFeding of What Happens. Harcourt Brace and Co., New York.

Davis, M. 1992. The amygdala and conditioned fear. In The Amygdala: Neurobiological Aspects of Emotion, Memory and Mental Dysfunction (J . P. Aggleton, Ed.), pp. 255-306. Wiley-Liss, New York.

Fanselow, M. S., and LeDoux, J. E. 1999. Why we think plasticity underlying Pavlovian fear conditioning occurs in the basolateral amygdala. Neuron 23: 229-232.

Friston, K. J ., Holmes, A. P., Worsley, K.J ., Poline, J .-P., Frith, C. D., and Frackowiak, R. S. J. 1995. Statistical parametric maps in functional imaging: A general linear approach. Hum. Brain Mapp. 2: $189-210$.

Haxby, J . V., Horvitz, B., Ungerleider, L. G., Maisog, J . M., Pietrini, P., and Grady, C. L. 1994. The functional organization of human extrastriate cortex: A PET-rCBF study of selective attention to faces and locations. J. Neurosci. 14: 6336-6353.

J osephs, O., Turner, R., and Friston, K. J . 1997. Event-related fMRI . Hum. Brain Map. 5: 243-248.

Konorski, J . 1967. Integrative Activity of the Brain: An Interdisciplinary Approach. Univ. of Chicago Press, Chicago.

LaBar, K. S., Gatenby, J . C., Gore, J . C., LeDoux, J . E., and Phelps, E. A. 1998. Human amygdala activation during conditioned fear acquisition and extinction: A mixed trial study. Neuron 20: 937945.

LeDoux, J. E. 1995. In search of an emotional system in the brain: Leaping from fear to emotion and consciousness. In The Cognitive Neurosciences (M. S. Gazzaniga, Ed.), pp. 1049-1061. MIT Press, Cambridge, MA.

Macknik, S. L., and Livingstone, M. S. 1998. Neuronal correlates of visibility and invisibility in the primate visual system. Nat. Neurosci. 1: 144-149.

Mcl ntosh, A. R., Cabeza, R. E., and Lobaugh, N. J . 1998. Analysis of neural interactions explains the activation of occipital cortex by an auditory stimulus. J . Neurophysiol. 80: 2790-2796.

Morris, J . S., Friston, K. J ., Buechal, C., Frith, C. D., Young, A. W., Calder, A. J ., and Dolan, R. J . 1998a. A neuromodulatory role for the human amygdala in processing emotional facial expressions. Brain 121: 47-57.

Morris, J. S., Friston, K. J ., and Dolan, R. J . 1998b. Experiencedependent modulation of tonotopic neural responses in human auditory cortex. Proc. R. Soc. London Ser. B 265: 649- 657.

Morris, J. S., Öhman, A., and Dolan, R. J. 1998c. Conscious and 
unconscious learning in the human amygdala. Nature 393: 467470.

Ohman, A., and Dimberg, U. 1978. Facial expressions as conditioned stimuli for electrodermal responses: A case of "preparedness"? J . Pers. Soc. Psychol. 36: 1251-1258.

Ohman, A., Flykt, A., and Lundqvist, D. 2000. Unconscious emotion: Evolutionary perspectives, psychophysiological data and neuropsychological mechanisms. In Cognitive Neuroscience of Emotion (R. D. Lane and L. Nadel, Eds.), pp. 296-329. Oxford Univ. Press, New York.

Pitkanen, A., Savander, V., and LeDoux, J . E. 1997. Organization of intra-amygdaloid circuitries in the rat: An emerging framework for understanding functions of the amygdala. Trends Neurosci. 20: 517-523.
Wagner, A. R. 1979. Habituation and memory. In Mechanisms of Learning and Motivation (A. Dickenson and R. A. Boakes, Eds.). Erlbaum, Hillsdale, NJ .

Weinberger, N. M. 1995. Retuning the brain by fear conditioning. In The Cognitive Neurosciences (M. S. Gazzaniga, Ed.), pp. 10711089. MIT Press, Cambridge, MA.

Whalen, P. J., Rauch, S. L., Etcoff, N. L., Mclnerney, S. C., Lee, M. B., and J enike, M. A. 1998. Masked presentations of emotional facial expressions modulate amygdala activity without explicit knowledge. J . Neurosci. 18: 411-418.

Worsley, K. J ., Marrett, P., Neelin, A. C., Friston, K. J ., and Evans, A. C. 1996. A unified statistical approach for determining significant signals in images of cerebral activation. Hum. Brain Mapp. 4: 58-73. 